\title{
Clues from star-formation histories - does the formation scenario of S0 galaxies depend on their luminosities?
}

\author{
Sudhanshu Barway ${ }^{1}$, Yogesh Wadadekar ${ }^{2}$, Kaustubh Vaghmare $^{3}$ \\ and Ajit Kembhavi ${ }^{3}$ \\ ${ }^{1}$ South African Astronomical Observatory, P.O. Box 9, 7935, Observatory, Cape Town, South \\ Africa; email: barway@saao.ac.za \\ ${ }^{2}$ National Centre for Radio Astrophysics, Post Bag 3, Ganeshkhind, Pune 411007, India \\ ${ }^{3}$ Inter University Centre for Astronomy and Astrophysics, Post Bag 4, Ganeshkhind, Pune \\ 411007, India
}

\begin{abstract}
Lenticular (S0) galaxies are a morphological transition class between ellipticals and early-type spirals. Recent studies indicate that the formation scenario for lenticular galaxies depends on their near-IR luminosity (Barway et al. 2007;2009;2011). Low-luminosity lenticular galaxies likely formed by the stripping of gas from the disc of late-type spiral galaxies, which in turn formed their pseudo bulges through secular evolution processes, possibly involving multiple episodes of star formation. On the other hand, more luminous lenticulars likely formed at early epochs through a rapid collapse followed by rapid star formation, similar to the formation process of elliptical galaxies, and are characterized by a old coeval stellar population in the bulge. To investigate the imprint of these distinct formation scenarios on stellar populations, we combine GALEX/SDSS/2MASS photometry to construct color-color diagrams for a carefully defined sample of 400 lenticular galaxies. We divide the sample into bright and faint groups as suggested by Barway et al. (2007). We find the (FUV-NUV) versus (NUV-K) color-color diagram shows larger scatter for faint lenticular galaxies while bright lenticular galaxies show a much tighter relation similar to that of ellipticals. Simple Stellar Population (SSP) analysis shows that ellipticals and bright lenticulars are dominated by a stellar population of age $>10^{9}$ years while faint lenticular galaxies are as young as $10^{8}$ years, providing evidence for relatively recent star formation activity. Further, if we use the (NUV-r) $<5.4$ color criterion to identify galaxies with recent (within $500 \mathrm{Myr}$ ) star formation, most faint lenticular galaxies fall into this category; bright lenticulars are mostly quiescent. Most faint lenticular galaxies generally have lower velocity dispersions. If we consider velocity as a virial mass indicator, majority of faint, recently star forming, lenticular galaxies have low virial masses compared to brighter lenticular galaxies. Such systematic differences in star formation history between bright and faint lenticular galaxies are consistent with the expectations from the luminosity dependent formation scenario described above.
\end{abstract}

Keywords. galaxies: elliptical and lenticular, cD — galaxies: evolution - galaxies: stellar content — infrared: galaxies — ultraviolet: galaxies

\section{References}

Barway S., Kembhavi A., Wadadekar Y. et al. 2007, ApJ, 661, L37

Barway, S., Wadadekar, Y., Kembhavi, A. K. et al. 2009, MNRAS, 394, 1991

Barway, S., Wadadekar, Y., \& Kembhavi, A. K. 2011, MNRAS, 410, L18 